\title{
INDIUM DOPING OF CdTe GROWN BY MOLECULAR BEAM EPITAXY*
}

\author{
G. Karczewski, A. Zakrzewski, M. Kutrowski, J. JaroszyńsKi, \\ W. Dobrowolski, E. Grodzicka, E. Janik, T. WoJTowicz, J. Kossut \\ Institute of Physics, Polish Academy of Sciences \\ Al. Lotników 32/46, 02-668 Warszawa, Poland
}

\section{AND A. BARCZ}

Institute of Electron Technology, Al. Lotników 32/46, 02-668 Warszawa, Poland

We report on $n$-type indium doping of CdTe films grown by molecular beam epitaxy on (001) GaAs substrates. By adjusting the flux of In atoms we can precisely control the carrier concentration over three orders of magnitude - from $8 \times 10^{14}$ up to $1.3 \times 10^{18} \mathrm{~cm}^{-3}$. In agreement with earlier reports we confirmed that $\mathrm{Cd}$ overpressure plays an important role in the doping process. The doping appears to be most effective for $\mathrm{Cd} / \mathrm{Te}$ pressure ratio of 1.5. For this value of $\mathrm{Cd} / \mathrm{Te}$ pressure ratio essentially $100 \%$ efficiency of doping is achieved at low In concentrations $\left(<10^{18} \mathrm{~cm}^{-3}\right)$. At higher In concentrations acceptor impurities compensate shallow donors limiting the concentration of free carriers.

PACS numbers: 68.55.Ln, 73.61.Ga

Although molecular beam epitaxy (MBE) is now commonly recognized as a standard method for growth of high quality CdTe films, considerable attention has still to be directed towards the study of doping of this material. Most of earlier investigations were focused on doping of CdTe grown on such substrates as: $\mathrm{CdTe}[1], \mathrm{Cd}_{0.96} \mathrm{Zn}_{0.04} \mathrm{Te}[2,3]$, and $\mathrm{InSb}$ [4], i.e., on substrates which are closely lattice matched to CdTe. In the case of CdTe films grown on GaAs, their crystal lattices are highly mismatched ( $14 \%$ mismatch). High quality of GaAs substrates and their relatively low price make, however, the $\mathrm{CdTe} / \mathrm{GaAs}$ system very attractive from the point of view of device applications.

In order to exploit the potential of CdTe for II-VI devices integrated with III-V semiconductors, good control of doping of MBE grown CdTe films is essential. In this investigation we focused on indium doping of (001) CdTe epilayers grown on (001) GaAs substrates. We choose indium as an $n$-type dopant, because of its low reactivity and its ready availability in high-purity elemental form, which makes this element ideal for doping in most of the crystal growth methods, and

${ }^{*}$ This work is supported in part by the State Committee for Scientific Research (Republic of Poland) through grant PBZ-ZO11/P4/93/01. 
especially in MBE. The main purpose of this investigation was to determine how electrical and optical properties of CdTe:In films are affected by growth conditions of $\mathrm{MBE}$ - in particular, by the In flux and the $\mathrm{Cd} / \mathrm{Te}$ pressure ratio.

The In-doped CdTe epilayers were grown on both, semi-insulating and highly $n$-doped GaAs substrates. The doping level was controlled by both, the flux of In atoms and the $\mathrm{Cd} / \mathrm{Te}$ beam pressure ratio. The substrate temperature was kept at about $250^{\circ} \mathrm{C}$. The source materials were $\mathrm{CdTe}$ and metallic Cd, both of $7 \mathrm{~N}$ purity. In order to unify the influence of the lattice mismatch all the investigated CdTe:In epilayers were of the same thickness, $d=3.5 \mu \mathrm{m}$. The techniques of characterization include low temperature photoluminescence, resistivity, and the Hall effect measurements. Concentrations of In atoms and their profiles were deduced from secondary ion mass spectroscopy (SIMS).

For quick, qualitative testing of effectiveness of the In-doping we applied low-temperature photoluminescence (PL). A typical PL spectrum of a doped epilayer is dominated by the neutral donor-bound exciton line $\left(\mathrm{D}^{0} \mathrm{X}\right)$ at $1.593 \mathrm{eV}$. The intensity of the $\mathrm{D}^{0} \mathrm{X}$-line strongly increases with increasing In flux, indicating a significant increase in the shallow donor concentration introduced into the $\mathrm{CdTe}$ lattice by the In dopants. In addition, from energies of zero-phonon and phonon replicas of the donor-acceptor pair recombination we derived the energy of the longitudinal optical (LO) phonon, $E_{\mathrm{LO}}=21 \mathrm{meV}$.

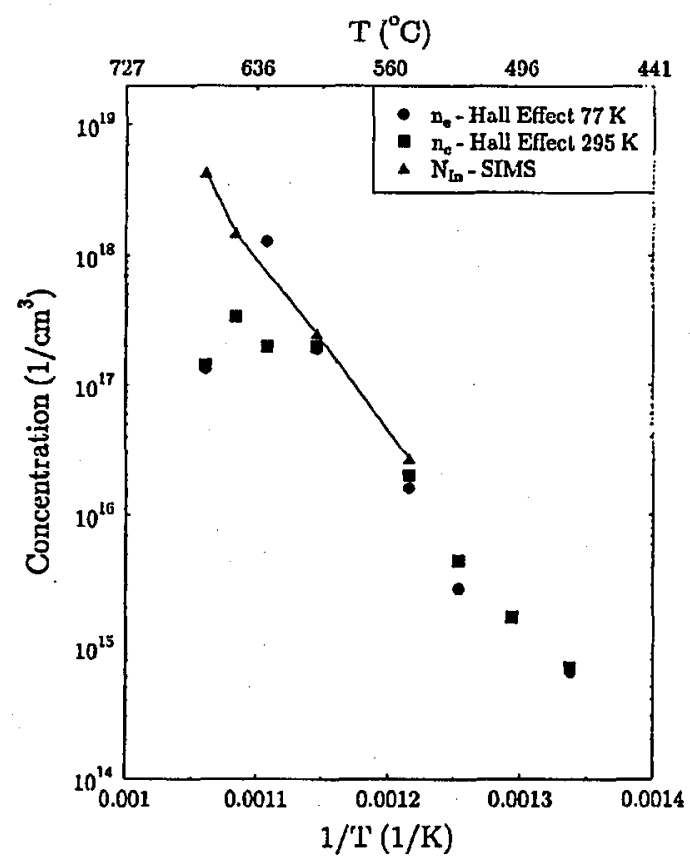

Fig. 1. Carrier concentration determined from the Hall effect measurements and concentration of indium atoms measured by SIMS as a function of inverse temperature of In cell, $1 / T_{\text {In }}$. 
Figure 1 shows free carrier concentration determined from the IIall effect measurements and concentration of indium atoms measured by SIMS as a function of invert temperature of In cell, $1 / T_{\text {In }}$, which corresponds to different In fluxes. All the samples were grown using the same $\mathrm{Cd} / \mathrm{Te}$ pressure ratio $(\mathrm{Cd} / \mathrm{Te}=1.5)$. By adjusting $T_{\text {In }}$ we are able to control effectively the carrier concentration in a wide range - from $8 \times 10^{14}$ up to $1.5 \times 10^{18} \mathrm{~cm}^{-3}$. For low In flux the concentrations of free carriers and In atoms are identical within experimental error, which indicates that the activation efficiency of the In-doping process is close to $100 \%$. For higher fluxes, when the concentration of In atoms exceeds the level of $10^{18}$, the Hall concentration significantly decreases, which points to an onset of strong compensation with self-activated acceptors. In addition,. SIMS provides evidence that In atoms are uniformly distributed in the films, which confirms earlier results that In atoms in $\mathrm{CdTe}$ are well localized and their migration is negligible at growth temperatures used by us [2].

Typical values of the mobility in our CdTe:In films are in the range of $200-800 \mathrm{~cm}^{2} /(\mathrm{V} \mathrm{s})$ at room temperatures. The mobility appears to be only slightly dependent on the electron concentration. The maximum value is reached for $n=$ $2 \times 10^{16} \mathrm{~cm}^{-3}$. The weak dependence of the mobility results in a proportionality between the conductivity of the films and the doping level. We established experimentally that the proportionality holds for over three orders of magnitude. Instead of the carrier concentration one can then discuss resistivity of the film, which can be measured much more precisely.

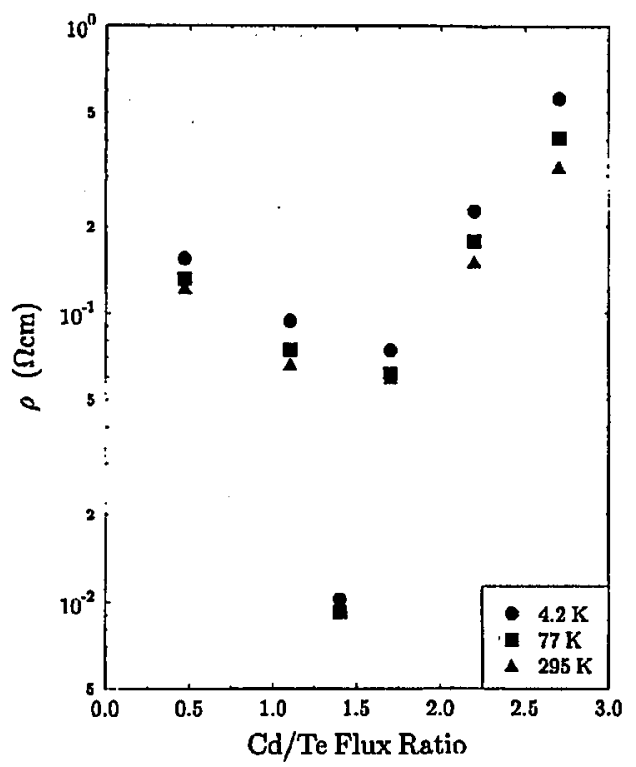

Fig. 2. Resistivity of CdTe epilayers as a function of the $\mathrm{Cd} / \mathrm{Te}$ pressure ratio. The layers were grown using identical In fluxes $\left(T_{\mathrm{In}}=630^{\circ} \mathrm{C}\right)$, and at the same substrate temperature, $T_{\mathrm{s}}=250^{\circ} \mathrm{C}$. 
From the analysis of resistivity data we estimated the activation energy of the shallow donors, $E_{\mathrm{d}}=22 \mathrm{meV}$, and the range of the $\mathrm{Cd} / \mathrm{Te}$ pressure ratio which yields optimum doping. In Fig. 2 we plotted resistivity of CdTe epilayers grown using fixed CdTe and In fluxes $\left(p_{\mathrm{CdTe}}=10^{-6}\right.$ torr, $\left.T_{\mathrm{In}}=630^{\circ} \mathrm{C}\right)$, but with different Cd fluxes, which corresponds to different values of $\mathrm{Cd} / \mathrm{Te}$ pressure ratio. It is interesting to notice that the lowest resistivity is reached for a considerable $\mathrm{Cd}$ overpressure, i.e. for the $\mathrm{Cd} / \mathrm{Te}$ ratio of 1.5 . Any deviation of the $\mathrm{Cd} / \mathrm{Te}$ pressure ratio from the optimal value results, however, in a significant increase in the resistivity. This result indicates that improper Cd pressure during the MBE process can considerably reduce the effective number of In atoms which contribute to the carrier concentration, i.e. it can decrease activation efficiency of In-doping in agreement with previous results $[2,3]$.

In summary, we demonstrated that uniform In doping of CdTe grown on strongly mismatched GaAs substrates were achieved under excess Cd flux. Carrier concentration can be effectively controlled by adjusting the In flux. In wide concentration range the activation efficiency of In doping is close to $100 \%$. Because of compensation by acceptor impurities, for high indium fluxes the effective number of indium atoms which contribute to the free carriers concentration significantly decreases.

\section{References}

[1] Y.S. Wu, A. Waag, R.N. Bicknell-Tassius, Appl. Phys. Lett. 57, 1754 (1990).

[2] F. Bassani, F. Tatarenko, K. Saminadayar, N. Magnea, A. Tardot, C. Grattepain, J. Appl. Phys. 72, 2927 (1992).

[3] S. Tatarenko, F. Bassani, J. Cryst. Growth 127, 318 (1993).

[4] D. Ashenford, P. Devine, J.H.C. Hogg, B. Lunn, C.G. Scott, J. Phys., Condens. Matter 3, S254 (1991). 